\title{
Nutritional status and COVID-19: an opportunity for lasting change?
}

\author{
Author: Shameer Mehta ${ }^{\mathrm{A}}$
}

The coronavirus disease of 2019 (COVID-19) pandemic has placed many healthcare systems, including the NHS, under unprecedented pressure. Mortality appears to be highest among older people and those with comorbidities, who are also often the most at risk of undernutrition in society. Despite international efforts to identify a specific treatment, therapy remains supportive and is principally focused on optimising respiratory function. However, the timely identification and correction of undernutrition also has the potential to improve outcomes cost-effectively, and should not be forgotten. This piece outlines why nutritional status may be particularly compromised during this crisis, among both the population and hospital inpatients. Practical steps to improve nutritional status at a time when hospital services are particularly stretched are also considered. Finally, the case is made for behaviour change at all levels including government, the general population and healthcare professionals.

KEYWORDS: Nutrition, screening, COVID-19, coronavirus

DOI: $10.7861 /$ clinmed.2020-0187

\section{Introduction}

On 11 March 2020, the World Health Organization (WHO) declared coronavirus disease 2019 (COVID-19) a pandemic.? As of 8 April 2020 there have been 1,484,811 cases worldwide, with 60,733 cases and 7,097 hospital deaths in the UK alone. ${ }^{2}$ While several clinical trials are underway in an attempt to identify specific therapies, current management remains largely supportive. The majority of deaths have involved older, frail and/or comorbid patients, ${ }^{3}$ groups who also have high rates of malnutrition. ${ }^{4}$ Advice from the UK government and Public Health England (PHE) has correctly focused on transmission reduction, including handwashing and social distancing. One daily session of outdoor exercise is permitted. However, there is comparatively little mention of optimal diet or nutritional status for the general population. Additionally, for hospitalised patients, at a time of unprecedented pressure on the NHS, there is potential for usual

Author: Aconsultant gastroenterologist, University College London Hospitals NHS Foundation Trust, London, UK nutritional screening and assessment practices to be overlooked. In this piece, the potential importance of undernutrition and nutritional therapy in COVID-19 will be discussed, together with practical suggestions to adapt and embed nutritional care into current ward-based clinical practice.

\section{Parallels from other conditions}

Given the recency of the pandemic, there is currently no published evidence relating to the impact of nutritional status on COVID-19 severity. Three parallels can be drawn, however, from data relating to older or polymorbid patients. Firstly, malnutrition is likely to be common. Multicentre studies demonstrate that 23-60\% of older patients in acute care settings are malnourished, ${ }^{4}$ with several prospective studies reporting a rate of $40-50 \%$ among hospitalised polymorbid patients. 5 ,6 Secondly, malnutrition adversely affects clinical outcomes. A large observational study found that complications, length of stay and mortality were significantly worse in untreated, nutritionally at-risk inpatients than in inpatients who were not at risk. ${ }^{7}$ Thirdly, these adverse effects are likely to be modifiable. Multiple large randomised studies have demonstrated that older or polymorbid inpatients provided with nutritional support experience improved quality of life ${ }^{8}$ and reduced complications (including infectious complications) ${ }^{6,9}$ with no significant impact on cost. ${ }^{9}$ Previous large-scale viral outbreaks can also be considered. Predictors of mortality from influenza by multivariate analysis in a study of 1,345 patients included malnutrition, which demonstrated an odds ratio (OR 25.0) greater than that for virus type (OR 7.1) or pulmonary infiltrates on chest radiography (OR 6.0). ${ }^{10}$ Indeed, as the authors noted, of all the predictors, only malnutrition and pneumonia were amenable to medical intervention.

\section{Undernutrition and COVID-19}

There are specific reasons why the nutritional status of patients and the general population may be particularly at risk during the current COVID-19 outbreak. These can be classified into three phases. The first phase is societal. The UK government announced a national lockdown on 23 March 2020, with all but essential journeys prohibited. This has predictably led to the temporary closure of many businesses and a significant economic downturn. Many people have lost livelihoods or received reduced incomes during this period. Those hit hardest are likely to be from already financially vulnerable groups, who may turn to cheaper food items of lower nutritional value. Indeed, the initial stage of the crisis 
saw supermarket shelves devoid of such non-perishable items. Moreover, the lack of availability of fresh produce may significantly impact older people, who rely on delivery services or who can visit stores only infrequently. Food banks and charities have struggled to cope with increased demand from social groups who previously did not require their services. ${ }^{11}$ The short-term effects of missing meals include not only fatigue, a reduced immune function and an increased risk of communicable diseases, ${ }^{12}$ but also a higher rate of complications after these diseases (such as viral infections) lead to hospitalisation.

The second phase relates to the challenges faced once patients are admitted to general hospital wards. No specific data relating to malnutrition on admission in patients with COVID-19 are available. However, given the age and comorbidity of this patient cohort, the data presented above and the additional societal issues, it is likely that a significant proportion will be undernourished on presentation. While in hospital, many patients are unable to feed themselves adequately due to problems with chewing, physical disabilities, cognitive disturbance, psychosocial issues or frailty. Medical and nursing teams are overstretched for a number of reasons, including staff shortages (due to sickness or self-isolation), caseload, unfamiliar working patterns, time spent liaising with families unable to visit their loved ones or donning/ doffing personal protective equipment (PPE). Additionally, the focus of medical teams lies principally on urgent clinical (primarily respiratory) issues rather than nutritional factors. Furthermore, a number of staff members are being redeployed from specialties in which nutritional care is not embedded into routine practice. These factors may result in suboptimal support for patients during mealtimes, as well as inadequate nutritional optimisation. Dietetic support is invaluable in overcoming these challenges. However, reorganisation of services across hospital trusts has restricted dietetic support, with some offering remote screening. The adequacy of this is limited by the availability of paperless records and consistent documentation of nutritional status by medical and nursing teams. There is, therefore, considerable potential for the usual processes of nutritional screening, assessment and therapy to be lost at a time when they are badly needed. Finally, the use of non-invasive ventilation (NIV) presents another barrier to eating and nasogastric (NG) tube feeding. The presence of an NG tube can lead to mask leakage and gastric distension, which may impair diaphragmatic function. Indeed, it has previously been shown that energy-protein intake among patients with acute respiratory distress syndrome on NIV is suboptimal. ${ }^{13}$

The third phase of nutritional risk occurs when patients require admission to the intensive therapy unit (ITU). While the specifics of nutritional therapy on ITU are beyond the scope of this piece, the importance of preserving muscle mass and function during a catabolic state such as infective respiratory failure is worth highlighting.

\section{How can we adapt?}

Given these challenges, which strategies should be employed to optimise nutritional status? At a societal level, it is entirely appropriate that the UK government over recent weeks has focused on the reduction of transmission of SARS-CoV-2. However, as time has passed and with no imminent end to social distancing, there is an urgent need not to shift focus, but to include strong messaging on the importance of nutrition. There currently exists an unprecedented and unique advantage of a captive audience on a national scale that should be exploited. Clear advice on adequate calorie intake and an optimal diet to support immune function should be provided, eg a variety of fresh fruit and vegetables, unsaturated fats, complex carbohydrate and sufficient protein and vitamin intakes. The British Dietetic Association ${ }^{14}$ and the $\mathrm{WHO}^{15}$ have issued useful guidance, although it could be argued that these could be disseminated more widely. Numerous influential social media accounts offer recipes and practical suggestions to achieve these targets; proactively engaging with these to ensure consistent advice could produce a significant impact rapidly. This needs to be partnered with aid - both financial and practical - for the most disadvantaged of society, including those from lower socio-economic groups, in order to facilitate behaviour change. Ideally, nutritional advice should be partnered with guidance on strengthening exercises, given that this combination is required to preserve or build muscle mass. ${ }^{16}$ Now is the time for stakeholders such as the UK government, PHE, professional bodies and the WHO to develop educational programmes within the constraints of social distancing to disseminate knowledge to the population, patients and healthcare workers.

More can also be done within the pre-ITU hospital setting. It is imperative that nutritional screening is not lost at this time. Validated tools such as MUST ${ }^{17}$ or NRS-2002 ${ }^{18}$ can be used, although trusts may have locally developed scores suited to local processes. Importantly, screening should not be confined to underweight patients, since clinically relevant sarcopenia can be present in obese individuals. A simplified tool could be considered to aid teams to perform screening and basic assessments to support nutritional therapy when dietetic support is unavailable. One local example is the creation of a set of prompts which are automatically generated on electronic COVID-19 ward round entries. This includes a screening tool score, leading on to predefined plans based on nutritional risk together with oral intake as assessed by a single question: 'can the patient finish their meals?'. Subsequent actions include repeating 5 days later, introducing volunteer-assisted feeding, prescribing oral supplements and instituting enteral feeding or dietetic referral, depending on risk. Enteral feeding is commenced if supplements are not tolerated after 48 hours. Safeguards are in place relating to NG tube placement, refeeding risk and monitoring. Ideally this should be first completed within $24-48$ hours of admission. A simplified tool such as this may help to embed nutritional care into the practice of busy COVID-19 ward teams and strike a balance between pragmatism and safety. Medical and nursing students, together with catering staff, can play vital roles in assisted feeding.

Given the efficacy of the prone position in improving ventilation in the ITU setting, there has been significant interest in extending this manoeuvre to conscious patients on general wards. However, the prone position is typically adopted for up to 18 hours per day in ventilated patients, which presents some logistical challenges. Not only is it difficult for conscious patients to maintain this position for such a lengthy period, but frail patients will be unable to adopt the position at all. The prone position is also not favourable for allowing usual oral intake patterns. When performed, therefore, it is ideal to ensure that meals, hydration and snacks are not missed. The Intensive Care Society has suggested 30-120-minute cycles of position changes in conscious patients, ${ }^{19}$ which may be more feasible to implement. Enteral feeding tubes also present potential logistical challenges, although the prone position is not a contraindication to enteral nutrition (EN). ${ }^{20}$ Indeed, the limited 
literature suggests that EN is safe while prone, with similar residual gastric volumes (RGVs) observed as with the supine position. ${ }^{21}$ Recent international COVID-19-specific guidelines support EN in prone patients, but suggest temporary cessation of feed to allow NG tube aspiration prior to position change, together with continued 8-hourly measurements of RGVs. ${ }^{22}$ Local experience 'on the ground' also suggests that another potential challenge with enteral feeding lies with feeding pumps. Most pumps are currently prioritised for use in ITU, leaving a shortage on general wards. Bolus, rather than continuous, feeding is therefore employed, which can lead to higher rates of gastric feed intolerance. Where post-pyloric feeding is required, the placement of bedside nasojejunal tubes may prove to be particularly useful at a time when endoscopy services are limited.

Another controversial area of nutritional care relates to the NIV setting. There is limited evidence to support decision-making in this context, with consequent variation in practice. To avoid difficulties with NG tubes, patients should be offered short, regular breaks to allow eating and drinking. This may, however, require additional nursing assistance. Where NG tubes are necessary, NIV masks with ports for NG tubes can be used, although these are not universally available. Pressure dressings may also help to minimise leakage. The use of high-flow nasal oxygen has increased in recent years. This carries the advantage of allowing oral alimentation, although once again use is limited by availability. Ultimately an individualised approach is required, one which accounts for nutritional risk, oral intake, available resources and respiratory compromise. For example, in a well-nourished patient with significant respiratory failure, delaying $\mathrm{EN}$ in order to optimise the efficacy of NIV and avoid mechanical ventilation may be reasonable. This, however, should be balanced with the knowledge that the work of breathing can account for up to $25 \%$ of energy expenditure and that progressive energy deficit may contribute to respiratory demise. ${ }^{23}$

While early consideration of EN should be advocated in the appropriate setting, suitable measures should also be taken to minimise risks arising from enteral tube placements. These procedures carry potential for aerosol generation, meaning that correct PPE must be worn. Additionally, continued vigilance is required in relation to $N G$ tube position verification - a malpositioned NG tube remains a never event. There is a need, however, to limit chest radiographs where possible, due to capacity issues and risk of transmission. Therefore, in those patients in whom there is difficulty in obtaining a gastric aspirate for $\mathrm{pH}$ checking, further attempts should be made after an hour and/or following repositioning of the patient. While there has been some initial concern over potential transmission risk from gastric fluid, this remains unproven. Using parenteral nutrition (PN) as a firstline feeding route solely due to the putative enteral transmission risk should be discouraged. The clinical risks of PN (both peripheral and central), including catheter-related bloodstream infection and the non-use of a functioning gut in the absence of intestinal failure, are difficult to justify. Some patients will require supplemental PN, but only after attempts have been made to optimise EN tolerability. ${ }^{24}$

Crucially, nutritional therapy should continue after discharge, given that some pre-existing risk factors are likely to persist once patients are back in the community. Finally, in parallel with clinical care, it is important that data are collected relating to the nutritional status of COVID-19 patients on admission, the impact on outcomes and the effects of nutritional therapy.

\section{Conclusion}

As we contend with COVID-19, it is critical to ensure that the nutritional needs of the population are met and sustained, including those who are most vulnerable. Prevention, diagnosis and treatment of malnutrition must also be included in the routine management of COVID-19 inpatients. We as clinicians should adapt traditional nutritional care pathways to allow temporary mechanisms for pragmatic and safe decision-making. Important areas such as obesity, primary care strategies and ITU care have not been discussed here, but merit separate consideration.

This period could be looked upon as an opportunity. The onus is on those in authority to promote and facilitate a nutritional culture, on the population to achieve behaviour change and on healthcare professionals to embed nutritional care into routine practice. None of these measures are new or groundbreaking, but perhaps have not been at the forefront in recent years. If we are collectively able to implement and sustain these strategies during and after this pandemic, there may be at least one positive legacy of COVID-19. Necessity is, after all, the mother of (re)invention.

\section{References}

1 World Health Organization. WHO Director-General's opening remarks at the media briefing on COVID-19 - 11 March 2020. Geneva: WHO. www.who.int/dg/speeches/detail/who-directorgeneral-s-opening-remarks-at-the-media-briefing-on-covid-1911-march-2020 [Accessed 21 April 2020].

2 GOV.UK. Number of coronavirus (COVID-19) cases and risk in the UK. London: Department of Health and Social Care and Public Health England, 2020. www.gov.uk/guidance/coronavirus-covid19-information-for-the-public [Accessed 9 April 2020].

3 Zhou F, Yu T, Du R et al. Clinical course and risk factors for mortality of adult inpatients with COVID-19 in Wuhan, China: a retrospective cohort study. Lancet 2020;395:1054-62.

4 Agarwal E, Miller M, Yaxley A, Isenring E. Malnutrition in the elderly: a narrative review. Maturitas 2013;76:296-302.

5 Felder S, Lechtenboehmer C, Bally M et al. Association of nutritional risk and adverse medical outcomes across different medical inpatient populations. Nutrition 2015;31:1385-93.

6 Jie B, Jiang Z-M, Nolan MT et al. Impact of nutritional support on clinical outcome in patients at nutritional risk: a multicenter, prospective cohort study in Baltimore and Beijing teaching hospitals. Nutrition 2010;26:1088-93.

7 Sorensen J, Kondrup J, Prokopowicz J et al. EuroOOPS: an international, multicentre study to implement nutritional risk screening and evaluate clinical outcome. Clin Nutr 2008;27:340-9.

8 Starke J, Schneider H, Alteheld B, Stehle P, Meier R. Short-term individual nutritional care as part of routine clinical setting improves outcome and quality of life in malnourished medical patients. Clin Nutr 2011;30:194-201.

9 Rypkema G, Adang E, Dicke $\mathrm{H}$ et al. Cost-effectiveness of an interdisciplinary intervention in geriatric inpatients to prevent malnutrition. J Nutr Health Aging 2004;8:122-7.

10 Maruyama T, Fujisawa T, Suga S et al. Outcomes and prognostic features of patients with influenza requiring hospitalization and receiving early antiviral therapy: a prospective multicenter cohort study. Chest 2016;149:526-34.

11 Gavron N. Coronavirus has made London's food banks even more important. OnLondon, 8 April 2020. www.onlondon.co.uk/nickygavron-coronavirus-has-made-londons-food-banks-even-moreimportant/ [Accessed 9 April 2020].

12 Dunn CG, Kenney E, Fleischhacker SE, Bleich SN. Feeding lowincome children during the Covid-19 pandemic. N Engl ] Med 2020;NEJMp2005638. 
13 Reeves A, White H, Sosnowski K, Tran K, Jones M, Palmer M. Energy and protein intakes of 545 hospitalised patients with acute respiratory failure receiving non-invasive ventilation. Clin Nutr 2014;33:1068-73.

14 British Diatetic Association. COVID-19 / coronavirus - advice for the general public. www.bda.uk.com/resource/covid-19-coronavirus-advice-for-the-general-public.html [Accessed 9 April 2020].

15 World Health Organization. Food and nutrition during selfquarantine: what to choose and how to eat healthily. Geneva: WHO, 27 March 2020. www.euro.who.int/en/health-topics/healthemergencies/coronavirus-covid-19/news/news/2020/3/foodand-nutrition-during-self-quarantine-what-to-choose-and-howto-eat-healthily [Accessed 9 April].

16 Rolland Y, Onder G, Morley JE, Gillette-Guyonet S, Abellan van Kan $\mathrm{G}$, Vellas B. Current and future pharmacologic treatment of sarcopenia. Clin Geriatr Med 2011:27;423-47.

17 British Association of Parenteral and Enteral Nutrition. Malnutrition Screening Tool - 'MUST' calculator. Redditch, UK: BAPEN. www. bapen.org.uk/screening-and-must/must-calculator. [Accessed 9 April 2020].

18 MD+Calc. Nutrition Risk Screening 2002 (NRS-2002). www.mdcalc. com/nutrition-risk-screening-2002-nrs-2002 [Accessed 9 April 2020].

19 Bamford P, Bentley A, Dean J, Whitmore D, Wilson-Baig N. ICS guidance for prone positioning of the conscious COVID patient 2020. London: Intensive Care Society, 2020. https://emcrit.org/
wp-content/uploads/2020/04/2020-04-12-Guidance-for-consciousproning.pdf [Accessed 9 April 2020].

20 Singer P, Blaser AR, Berger MM et al. ESPEN guideline on clinical nutrition in the intensive care unit. Clin Nutr 2019;38:48-79.

21 Linn DD, Beckett RD, Foellinger K. Administration of enteral nutrition to adult patients in the prone position. Intensive Crit Care Nurs 2015;31:38-43.

22 Chapple L-A, Fetterplace K, Ridley E. Nutrition management for critically and acutely unwell hospitalised patients with COVID-19 in Australia and New Zealand. Mornington, Victoria: AuSPEN, 6 April 2020. https://custom.cvent.com/ FE8ADE3646EB4896BCEA8239F12DC577/files/93ecb5eadf7244faa98d9848921428a8.pdf [Accessed 21 April 2020].

23 Singer $P$, Rattanachaiwong $S$. To eat or to breathe? The answer is both! Nutritional management during noninvasive ventilation. Crit Care 2018;22:27.

24 Barazzoni R, Bischoff SC, Breda ] et al. ESPEN expert statements and practical guidance for nutritional management of individuals with SARS-CoV-2 infection. Clin Nutr 2020;j.clnu.2020.03.022.

Address for correspondence: Dr Shameer Mehta, University College London Hospitals NHS Foundation Trust, 250 Euston Road, London NW1 2PG, UK.

Email: shameer.mehta@nhs.net

\title{
Management and care of tracheostomised patients with prolonged disorders of consciousness (PDOC) during COVID-19
}

\author{
This guidance, which supplements the PDOC national clinical \\ guidelines published in March 2020, supports decision-making \\ in tracheostomised patients with PDOC and takes into account \\ the requirements to protect patients and staff from the risks of \\ acquiring COVID-19.
}

Download the guidelines: www.rcplondon.ac.uk/pdoc-covid-19 